\title{
Bioinspired Low-Frequency Material Characterisation
}

\author{
Robin C. Rainier, ${ }^{1}$ S. Assous, ${ }^{1}$ P. B. Wilkinson, ${ }^{2}$ D. A. Gunn, ${ }^{2}$ P. D. Jackson, ${ }^{2}$ J. G. Rees, ${ }^{2}$ \\ R. L. O'Leary, ${ }^{3}$ and M. A. Lovell ${ }^{1}$ \\ ${ }^{1}$ Department of Geology, University of Leicester, University Road, Leicester LE1 7RH, UK \\ ${ }^{2}$ British Geological Survey, Kingsley Dunham Centre, Keyworth, Nottingham NG12 5GG, UK \\ ${ }^{3}$ Department of Electronic and Electrical Engineering, Centre for Ultrasonic Engineering, 204 George Street, Glasgow G1 1XW, UK
}

Correspondence should be addressed to S. Assous, sa251@le.ac.uk

Received 31 May 2011; Revised 16 January 2012; Accepted 25 January 2012

Academic Editor: Gurvinder Virk

Copyright ( 2012 Robin C. Rainier et al. This is an open access article distributed under the Creative Commons Attribution License, which permits unrestricted use, distribution, and reproduction in any medium, provided the original work is properly cited.

\begin{abstract}
New-coded signals, transmitted by high-sensitivity broadband transducers in the $40-200 \mathrm{kHz}$ range, allow subwavelength material discrimination and thickness determination of polypropylene, polyvinylchloride, and brass samples. Frequency domain spectra enable simultaneous measurement of material properties including longitudinal sound velocity and the attenuation constant as well as thickness measurements. Laboratory test measurements agree well with model results, with sound velocity prediction errors of less than $1 \%$, and thickness discrimination of at least wavelength/15. The resolution of these measurements has only been matched in the past through methods that utilise higher frequencies. The ability to obtain the same resolution using low frequencies has many advantages, particularly when dealing with highly attenuating materials. This approach differs significantly from past biomimetic approaches where actual or simulated animal signals have been used and consequently has the potential for application in a range of fields where both improved penetration and high resolution are required, such as nondestructive testing and evaluation, geophysics, and medical physics.
\end{abstract}

\section{Introduction}

Acoustic techniques are widely used in material characterisation, nondestructive testing evaluation (NDTE) [111], sonar applications [12-19], medical physics imaging [20] and geophysics [21], as they may be applied both noninvasively and nondestructively and are relatively simple to deploy. Underwater material testing is common as many solids are acoustically well matched with water, and no coupling medium is required [5]. Methods commonly utilised range from measuring time delay and amplitude of narrowband ultrasonic pulses propagated through materials [1] to use of broadband spectral techniques $[2,3]$. Frequencies in immersion applications are typically in the $0.5-12 \mathrm{MHz}$ range, with transducer central frequencies of 5 or $10 \mathrm{MHz}$ in cases where sample thickness is from $2 \mathrm{~mm}$ to $40 \mathrm{~mm}$ [1-4], thus allowing reflections from front and back faces to be separated in time $[1,3-5]$. Other applications utilise the interference spectra obtained when signals reflected from the front and back faces overlap in time $[6,7]$ or direct phase measurement [8]. Many of the principles also apply to air-coupled NDTE [9-11]; in particular, broadband capacitance devices are used in spectral measurement of material resonances [10]. Low-frequency acoustic testing of elastomeric materials for underwater applications is often performed using panel tests [12-19] in which applied frequencies are commonly from a few $\mathrm{kHz}$ to $50 \mathrm{kHz}[12-$ 15], but sometimes extended to $500 \mathrm{kHz}$ [16-19]. The target panels are typically thin with respect to wavelength, resulting in pulse overlap and interference spectra. Measurements of insertion loss and echo reduction are performed to determine longitudinal velocity, attenuation and impedance [12-19]; frequency bursts or broadband pulses are typically used [12, 14-18]. Parametric arrays are used in broadband measurements as they minimise interference from pulse overlap, edge diffraction, and boundary reflections within test tanks. Characterisation over broad frequency bands is, however, almost always achieved only by use of successively narrower frequency band scans $[13,19]$. 
When performing acoustic measurements, choice of ultrasonic frequency is subject to a trade-off between axial resolution and penetration; higher frequencies provide greater resolution but suffer more attenuation. In nature, bats and dolphins achieve greater resolution than would be achievable using engineered signals, yet live in highly attenuating media [22]. Consequently, they have been studied in relation to developing ultrasonic engineering applications [22]. Both animal groups echolocate using broadband signals, but deliver their energy in different ways because of their operating environments. For instance, dolphins live in water where they are acoustically well matched to their environment, whereas bats (mostly) live in air where the acoustic impedance mismatch and attenuation parameters are higher. In general, dolphins use short-duration, highintensity broadband signals, described as "clicks," which contain between 4 and 8 cycles over $40-70 \mu$ s, with a centre frequency of $100 \mathrm{kHz}$ or more [23]. They can resolve small changes in wall thickness of cylinders that are far less than one wavelength thick $[23,24]$. Also they can discriminate between steel, aluminium, and coral rock aggregates encased in degassed epoxy resin [25], detect 2.5 and $7.62 \mathrm{~cm}$ diameter water-filled spheres at distances of 70-100 m [23, 26], and detect buried objects [27]. In contrast, most bat species emit longer-duration, high-intensity, broadband, frequencymodulated (FM) or combined FM, and constant frequency signals, in pulses of 0.3 to $300 \mathrm{~ms}$, with frequencies from around 10 to $200 \mathrm{kHz}$ [28]. Although bats and dolphins employ different signal strategies, it is generally considered that they perceive the distance to objects from echo delay, and the shape of objects from the interference spectrum generated when multiple reflections from different parts of an object overlap [23].

A key development in acoustic signal processing has been the use of broadband signals such as FM chirp signals with pulse compression techniques to derive time of flight and amplitude information for imaging, as has been well demonstrated in air coupled NDTE $[10,11]$, high-frequency material characterisation [20], and marine sonar [21]. Properties of the FM signal, particularly linear FM (LFM), include good signal to noise ratio (SNR), autocorrelation properties, and delivery of high ultrasonic energy levels [10, 11, 21]; they have more robust ultrasound features than other coded waveforms [20]. The use of window functions (smoothing edges) has improved measurements using LFM chirps by reducing ripple distortion and rise and fall overshoots [10, 11]. Gan et al. [10] used LFM chirp signals modulated by a Hanning window for air-coupled ultrasonic imaging, providing improved SNR and autocorrelation characteristics over narrowband pulses [10]. Pallav et al. [11] used an elliptical Tukey window for chirp modulation in air coupled systems, giving improved performance over other windows. Misaridis and Jensen [20] concluded that LFM signals provide up to $10 \mathrm{~dB}$ gain in SNR over binary complementary Golay-coded waveforms for imaging in highly attenuating media; benefits are afforded by use of an amplitude tapering function such as a Tukey window. Chirp subbottom profilers are used in subsea geophysical imaging to generate repeatable 1 to $24 \mathrm{kHz}$ source signatures that are better controlled than boomer and pinger signals, resulting in higher resolution and penetration [21]. A broad bandwidth chirp with a squared taper function is commonly optimal for pulse compression techniques in subsea imaging [21]. Despite these advances, however, obtaining high-resolution measurements of material properties and thickness has remained a significant problem when using acoustic measurements in highly attenuating environments.

\section{Development of New Biologically Inspired Methodologies}

To address this problem, we present a method for characterising material properties, including thickness, sound velocity, and attenuation of materials using coded signals with better penetration/low frequencies, but gain high resolution by optimising the energy delivery transducer and signal design system. This is possible through the design and application of novel, biologically inspired signals. Details of signal design and modelling are outlined below, prior to a description of tests that demonstrate their successful application.

2.1. Signal Design. The coded signals are designed to use prolonged energy delivery, in a manner similar to that employed by bats, which combine multicycle components [23] (Figure 2(a)), yet the bandwidths are similar to those used by dolphins [23]. Our approach differs significantly from past biomimetic approaches, where actual or simulated animal signals have been used [22, 29, 30]. Our signals (Figure 2(b)), constructed by summing a series of sine waves in steps, governed by the required resolution, with a zero initial phase offset, are termed zero phase $(\mathrm{ZPH})$ signals (informally known as "ladder chirps"). The performance of these in terms of energy delivery can be compared with LFM upsweeps (Figure 2(a)), commonly used in material characterisation studies, that give a high SNR and a sharp main lobe in the autocorrelation function (ACF). The $\mathrm{ZPH}$ signals have an ACF comparable to that of LFM signals, but lower SNR. They appear to be useful alternatives to LFM signals as they provide concentrated energy in a short duration (similar to a dolphin signal). Furthermore, they do not have some of the analysis limitations associated with LFM signals — which are basically time-varying and inhibit the use of conventional tools such as the discrete Fourier transform (DFT) to correctly determine the spectral amplitude response. Figures 2(c) and 2(d) show the magnitude response of the two types of signals. It is clear that the response of the $\mathrm{ZPH}$ is much better than that of the LFM; this is because the ZPH signal contains an integer number of cycles at each frequency, enabling ready analysis of this signal using the DFT. Effectively, we are adapting the signal to the tool to improve amplitude, frequency, and phase estimation.

2.2. Calculating Theoretical Transmission and Reflection Spectra. Use of the coded signals in determining material properties, including longitudinal sound velocity, the attenuation constant, and thickness measurements, is explored through modelling. In this, and the calculation of theoretical 
transmission and reflection spectra, it is assumed that a plane acoustic pressure wave is incident in the $z$ direction, normal to the layer interfaces, and that the layers are infinite and homogeneous perpendicular to $z$. The $j$ th layer is characterised by the density $\rho_{j}$ and speed of sound $c_{j}$. The pressure in the $j$ th layer is given by

$$
p_{j}(z, t)=A_{j} e^{i\left(k_{j} z-\omega t\right)}+B_{j} e^{-i\left(k_{j} z+\omega t\right)},
$$

where $i=\sqrt{-1}, \omega$ is the angular frequency, the wavenumber is

$$
k_{j}=\frac{\omega}{c_{j}}+i \alpha_{j},
$$

and $\alpha_{j}$ is the attenuation coefficient in $\mathrm{Npm}^{-1}$. Following Mikeska and Behrens [17], the attenuation coefficient is assumed to be at most a quadratic function of frequency, $f$, having the form:

$$
\alpha(f)=a f+b f^{2} .
$$

The regions 0 and $n$ are assumed to be semi infinite, and the layers $1, \ldots, n-1$ have thicknesses $d_{1}, \ldots, d_{n-1}$, respectively. The transmission and reflection coefficients through the layered structure are calculated using a transfer matrix approach. The transfer matrix across the $j$ th layer is given by

$$
L_{j}=\left[\begin{array}{cc}
e^{-i k_{j} d_{j}} & 0 \\
0 & e^{i k_{j} d_{j}}
\end{array}\right] .
$$

The appropriate boundary conditions at the interfaces between layers are equality of pressure and normal particle velocity [31]. Applying these at the boundary between layers $j$ and $j+1$ yields, the transfer matrix across the $j$ th interface:

$$
I_{j}=\frac{1}{2}\left[\begin{array}{cc}
\left(1+\frac{\rho_{j} k_{j+1}}{\rho_{j+1} k_{j}}\right) & \left(1-\frac{\rho_{j} k_{j+1}}{\rho_{j+1} k_{j}}\right) \\
\left(1-\frac{\rho_{j} k_{j+1}}{\rho_{j+1} k_{j}}\right) & \left(1+\frac{\rho_{j} k_{j+1}}{\rho_{j+1} k_{j}}\right)
\end{array}\right] .
$$

The transfer matrix $\Pi$ for the whole structure is given by the product of the transfer matrices of the layers and their interfaces:

$$
\Pi=I_{0} L_{1} I_{1} L_{2} \ldots I_{n-1} .
$$

This transfer matrix relates the pressure wave in region 0 to that in region $n$ via the expression:

$$
\left[\begin{array}{c}
A_{0} e^{i k_{0} z_{0}} \\
B_{0} e^{-i k_{0} z_{0}}
\end{array}\right]=\Pi\left[\begin{array}{c}
A_{n} e^{i k_{n} z_{n-1}} \\
B_{n} e^{-i k_{n} z_{n-1}}
\end{array}\right] .
$$

Setting the incident condition $A_{0}=1$, noting that $B_{n}=0$, and assuming that $z_{0}=0$ yields the transmission coefficient:

$$
T=A_{n}=\frac{e^{-i k_{n} z_{n-1}}}{\Pi_{1,1}},
$$

and the reflection coefficient:

$$
R=B_{0}=\frac{\Pi_{2,1}}{\Pi_{1,1}} .
$$

The transfer matrix approach allows modelling of propagation through an arbitrary numbers of layers. It produces the same results as recursive application of the expressions for single-layer transmission and reflection coefficients [31], but it calculates the overall coefficients directly rather than iteratively. In this paper, the model used to fit the experimental data consists of one layer of test material between semi-infinite regions of water, in which case the single-layer expressions also give the same direct results. To fit the data, it is assumed that the density and thickness of the test material are known, as are the density and speed of sound of water. It is further assumed that the attenuation coefficient in water is negligible. The speed of sound is determined from the spacing of the resonances, $\Delta f$, in the transmission or reflection spectra of the thickest sample using

$$
\Delta f=\frac{c_{1}}{2 d_{1}} .
$$

Subsequently, the attenuation coefficient parameters $a_{1}$ and $b_{1}$ are found by curve fitting the modelled data to the experimental data for the thickest sample. These parameters are then used to produce modelled data for differing thicknesses of the same material.

\section{Experimental Testing}

To evaluate the effectiveness of LFM and ZPH signals in lowfrequency material characterization, tests were performed at the joint University of Leicester/British Geological Survey Ultrasonic Rig Facility located in Keyworth, Nottingham, UK. This comprises a water tank $(1.65 \mathrm{~m} \times 1.43 \mathrm{~m} \times$ $0.90 \mathrm{~m}$ ) with infrastructure for the precise mounting and positioning of transducers and target materials, housed in a temperature controlled laboratory with water temperature continually logged (Figure 1(a)). Custom built transducers (Alba Ultrasound Ltd., Glasgow, UK) comprise highsensitivity piezoelectric composite main elements having a $-6 \mathrm{~dB}$ fractional bandwidth approaching $100 \%$ around $100 \mathrm{kHz}$. The transducers operate in the rarely explored frequency range of $40-200 \mathrm{kHz}$ - an order of magnitude lower than commonly used in acoustic spectroscopy, but higher than those generally used in marine sonar. The motivation for using this frequency range is to readily emulate the echolocation systems of bats and dolphins and explore bioinspired applications.

Signals are generated and acquired using a highspecification modular system containing a ZT530PXI, 16bit arbitrary waveform generator and a ZT410PXI, 16bit digital storage oscilloscope (ZTEC Instruments Inc.). A C++ programme was developed to automate the signal transmission and reception procedure. An illustration of the setup is shown in Figure 1(b). Ultrasonic signals are propagated from a transmitting transducer $(\mathrm{Tx})$ to a receiving transducer $(\mathrm{Rx})$ set approximately $1 \mathrm{~m}$ apart and facing 


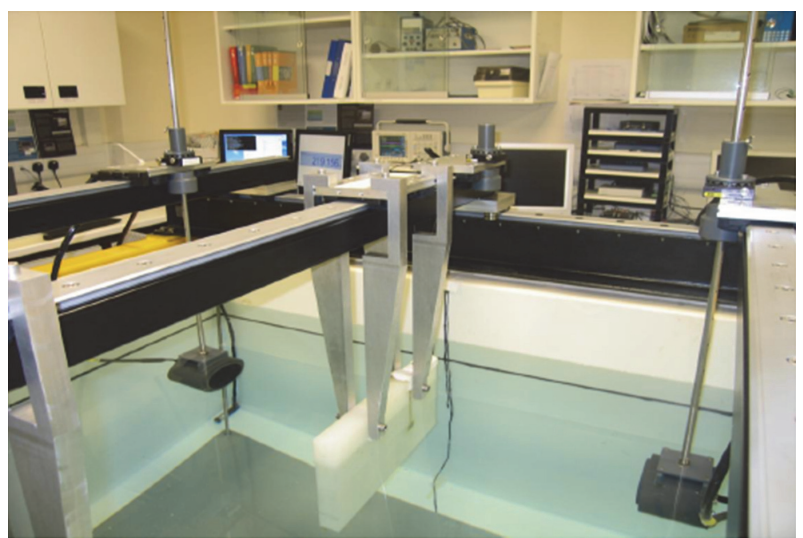

(a)

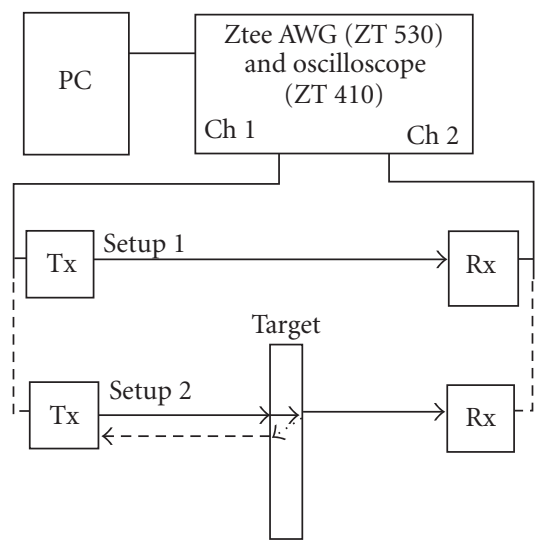

(b)

FIGURE 1: Experimental setup. (a) laboratory picture for the experimental set up. (b) diagram of experimental setup showing transmitting transducer $(\mathrm{Tx})$, receiving transducer $(\mathrm{Rx})$. Reflected and outgoing signals collected on channel 1 (Ch 1) of the oscilloscope, received signals collected on channel 2 (Ch 2). Computer (PC) used for controlling signal delivery and acquisition.

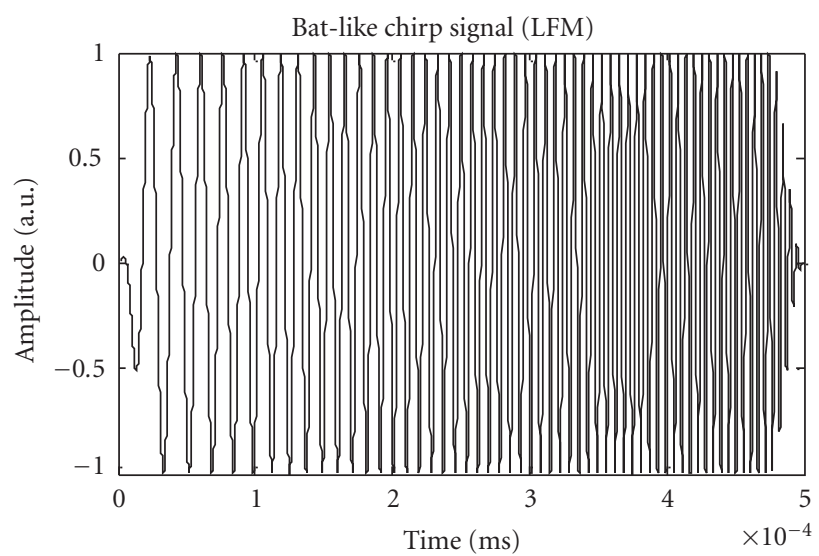

(a)

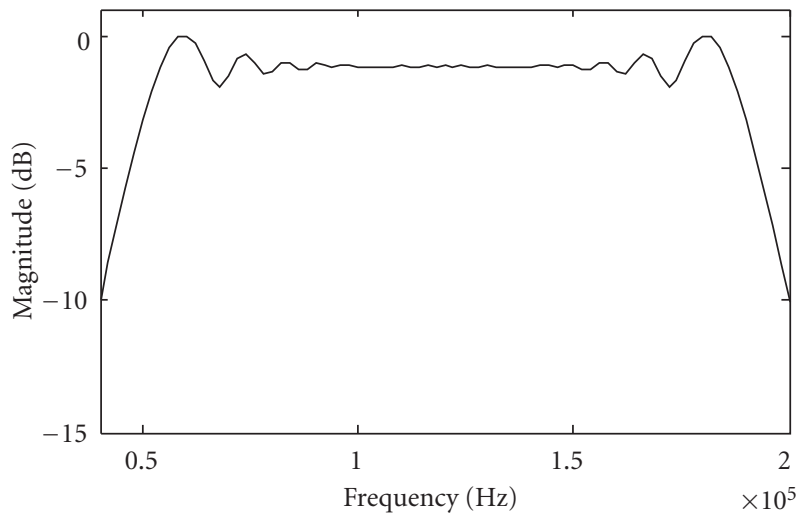

(c)

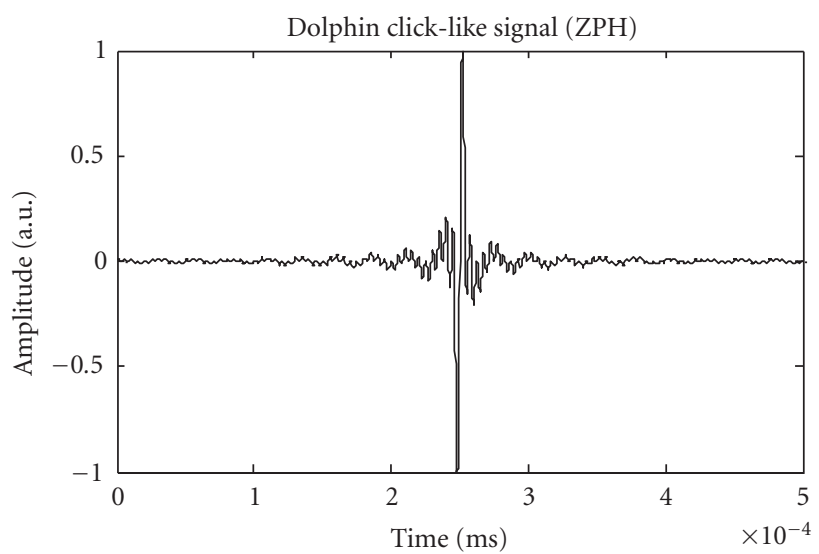

(b)

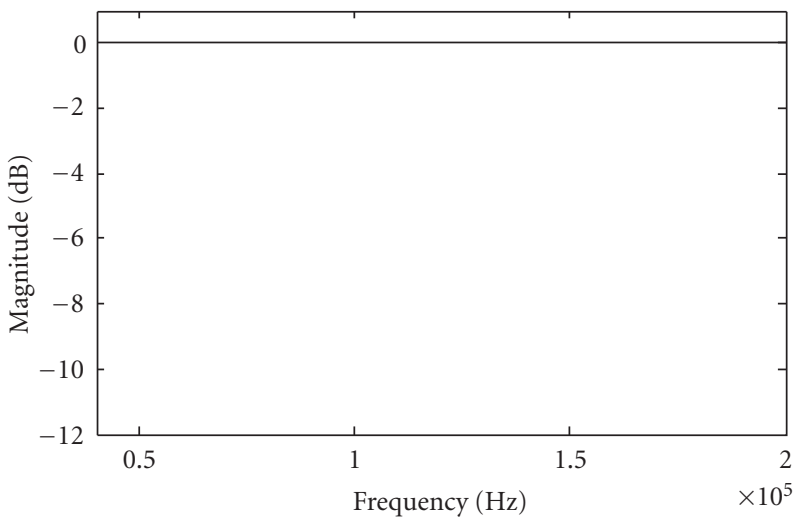

(d)

FIGURE 2: Synthetic signals developed for experimentation and their related frequency responses: (a) linear frequency-modulated (LFM) chirp signal, (b) zero phase (ZPH) ladder chirp signal, (c) magnitude response of (a), and (d) magnitude response of (b). 
each other. Signals are transmitted directly through water (mean temperature $20.2^{\circ} \mathrm{C}$ with a standard deviation of $0.3^{\circ} \mathrm{C}$ ) or also through a target, set centrally at a normal angle of incidence to the transducers; a reflected signal is also acquired when a target is in place. During tests, a reflection from the target is also collected on the transmitter; a reference signal is collected separately via a pulse/echo from the air-water interface, with the Tx placed on the bottom of the tank facing the surface. The setup differs to that used for measuring insertion loss (IL) and echo reduction (ER) in sonar applications [13], where IL is obtained by measuring the signal before and after placing a target in between the projector and hydrophone, and where ER is obtained by measuring the incident and reflected sound by a hydrophone placed between the projector and target. As measurements of IL and ER are made separately, alignment issues can affect the measurements [13]. In our setup, Tx may be used for transmitting and receiving reflections, thus transmission measurements can be made simultaneously; the transducer positions remain constant whilst the sample may be moved in and out of the ultrasonic propagation path, minimising alignment problems. The transducers are further from the target than the hydrophone, thus signal overlap is reduced. Extraneous reflections from tank components are not analysed as they arrive far later than the pulses of interest.

During testing data are acquired with a $10 \mathrm{MHz}$ sampling frequency, for 30000 points, using 32 averages. The transmitted, received, and reflected signal data are saved in binary format data and exported into Matlab for analysis. Spectral analysis is performed on signals using the DFT and evaluated using in-house Matlab code. The length $(N)$ and sampling frequency $\left(F_{s}\right)$ of signals are selected to ensure that the spectral resolution represented by the ratio $\left(F_{s} / N\right)$ should fit within predetermined frequency bins and avoid spectral leakage. The linear magnitude spectra are converted to decibels $(\mathrm{dB})$ by referencing the energy magnitude spectra through a target to the energy magnitude spectra through water. The reference spectra for transmitted signals are acquired for transmission through water, whereas for reflected signals, they are reflected off the air water interface, where the two-way travel time is kept the same. This processing step removes the effect of propagation through water and the frequency response of the transducer. Both ZPH and LFM signals, as described above, are used in the tests. Design recommendations for LFM signals from previous studies have included sweeping a band slightly larger than the passband of the transducer, with a hightime bandwidth product and using a window function [10, $11,20,21]$. Our $40-200 \mathrm{kHz}$ bandwidth LFM signal, which sweeps a band slightly larger than the $-6 \mathrm{~dB}$ range of our transducers, is approximately $70-170 \mathrm{kHz}$. The length of the signals is selected to have as large a time bandwidth product as possible, yet short enough to ensure no extraneous tank reverberation interference. The signals are also designed so that acquired signals can be analysed using the DFT with frequency binning. The signals have similar features to biological signals, but with well-constrained properties so that they can be analysed accurately using available signal processing tools. A 10\%-tapered Tukey window is applied to both LFM and ZPH signals. The window improved the signal characteristics and reduces the transducer "turn on" and "turn off" impulse responses. The signals (created with a $10 \mathrm{MHz}$ sampling frequency) are $500 \mu$ s length (to avoid spurious reflections), and $\mathrm{ZPH}$ signals are created with $2 \mathrm{kHz}$ steps. Data are collected using a $500 \mu$ s window, starting at the onset of the received or reflected signal, which allows $2 \mathrm{kHz}$-frequency resolution to be obtained. Transmission and reflection magnitude spectra are calculated as described above. Frequency domain spectra are calculated for the 40$200 \mathrm{kHz}$ range, with $2 \mathrm{kHz}$ resolution, by taking a 5000-point window of the acquired data.

In order to optimally use bioinspired signals with a bandwidth similar to that employed by bats and dolphins, we have selected targets that will allow discrimination of density at the appropriate frequencies. These include relatively homogeneous polypropylene (PP), polyvinylchloride (PVC), and brass panels of variable thickness $(0.5-40 \mathrm{~mm}$ thick, comparable to those used in previous panel tests-see above), but thin enough to make it impossible to separate front and back face reflections using echo delay techniques at the adopted wavelengths. These are determined instead using pulse overlap techniques and interference spectra from which material property information including longitudinal sound velocity, the attenuation constant, and prediction of thickness (via knowledge of the sound velocity) may be derived. As sound diffraction is a problem in lowfrequency measurement $[13-15,18]$, the rectangular $(300 \times$ $400 \mathrm{~mm}$ ) panels have lateral dimensions significantly greater than the wavelengths used $(7.5-37.5 \mathrm{~mm}$ for $40-200 \mathrm{kHz})$; consequently the whole beam remains within the panel boundaries.

\section{Results}

Examples of time domain LFM received signals for the transmission and pulse echo are shown in Figures 3(a) and 3(b). Examples of the normalised spectral response obtained by transmitting LFM and ZPH signals through water are shown in Figure 3(c). It can be seen that the received LFM and $\mathrm{ZPH}$ signals produce spectral responses that are almost identical, except at the extremes of the frequency range where slight differences are caused by low transducer sensitivity. Examples of the time domain pulse-echo received signals for different thicknesses of PVC and brass panels are shown in Figures 4(a) and 4(b). The spectral responses obtained for $40 \mathrm{~mm}$ thick panels in transmission and pulse echo (when LFM signals are applied) are shown in Figures 5(a) and 5(b), respectively. The spectral responses show periodic features the dimensions of which vary depending on material type. The transmission and pulse echo spectra differ in shape for different materials. The first maximum in the transmission spectrum (or minimum in the reflection spectrum) occurs at the fundamental frequency, where the thickness equals half the wavelength; subsequent maxima/minima occur at multiples of the fundamental frequency $[7,32]$. The peak spacing is greater for the brass panels than the plastic samples, indicating a higher sound velocity. The spectral shape is indicative of the mechanical $Q$ factor, which is 


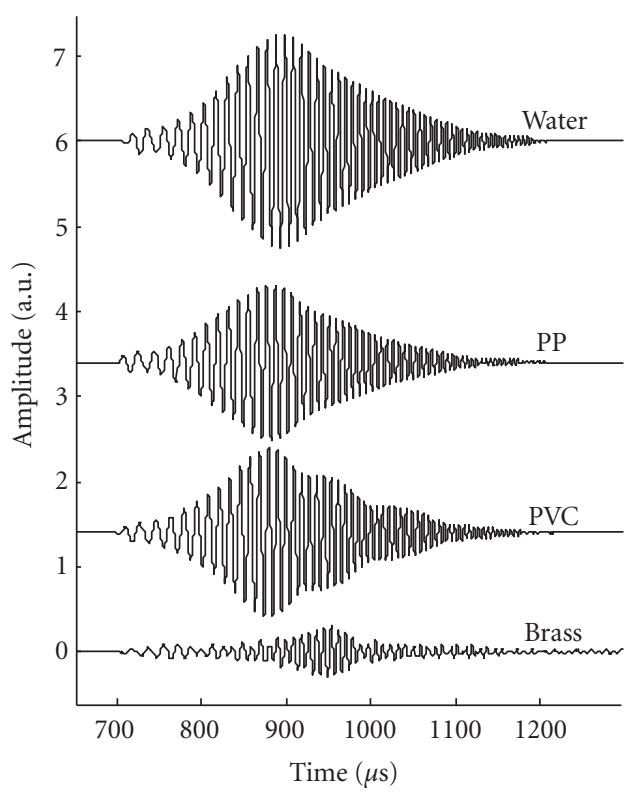

(a)

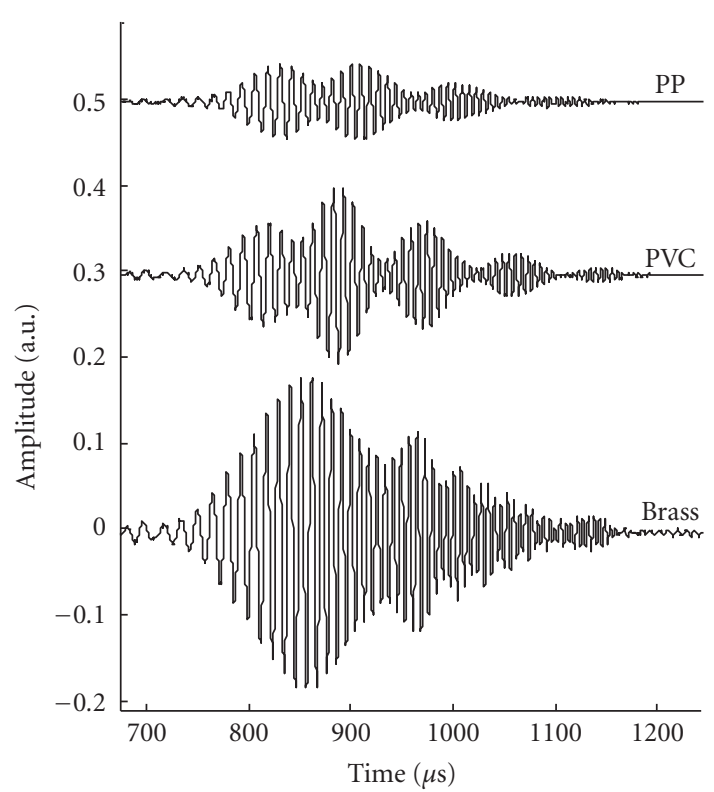

(b)

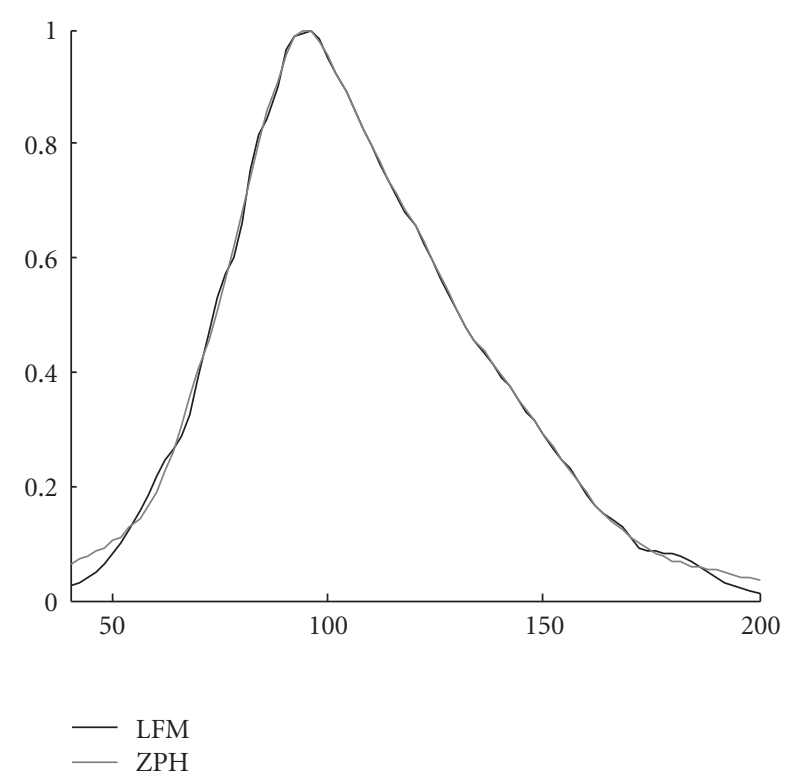

(c)

FIGURe 3: Time domain signals acquired for $40 \mathrm{~mm}$ thick polypropylene (PP), polyvinylchloride (PVC), and brass targets; (a) transmitted signals with water signal shown for reference, (b) reflected signals, and (c) Frequency spectra for the different signals received through water: normalised spectra for the linear frequency-modulated (LFM) chirp and zero phase (ZPH) ladder chirp.

related to attenuation by $\alpha=\pi f / Q v, Q_{\text {mech }}$, where $Q_{\text {mech }}=$ $f_{0} /\left(f_{1}-f_{2}\right)$, where $f_{0}$ is the mechanical resonance frequency, $f_{1}$ and $f_{2}$ are the frequencies where energy is half that at resonance, and $v$ is the velocity. The peaks are sharper for brass than for the plastics, indicating the higher $Q$ factor of the material. An underlying slope to the plot can be observed, in addition to the peaks, caused by attenuation-which is a function of frequency [17].

Examples of the received ZPH and LFM signals following transmission through $40 \mathrm{~mm} \mathrm{PVC}$ and pulse echo are shown in Figures 6(a) and 6(b), respectively. The spectral responses are almost identical, except in the bandwidth limits; this is because of decreased transducer sensitivity.

Test and modelled frequency spectra are shown in Figures 7, 8 and 9, for variable thickness PP, PVC, and brass panels, respectively. As noted, the peak spacing is dependent on the speed of sound in the material and its thickness; when material thickness increases the peak spacing decreases. The resonant features can be observed in panels of $10 \mathrm{~mm}$ thickness or greater; for thinner panels, the resonant spacing is larger than the bandwidth. The modelled and test results agree with PP and PVC panels. The test brass spectra 


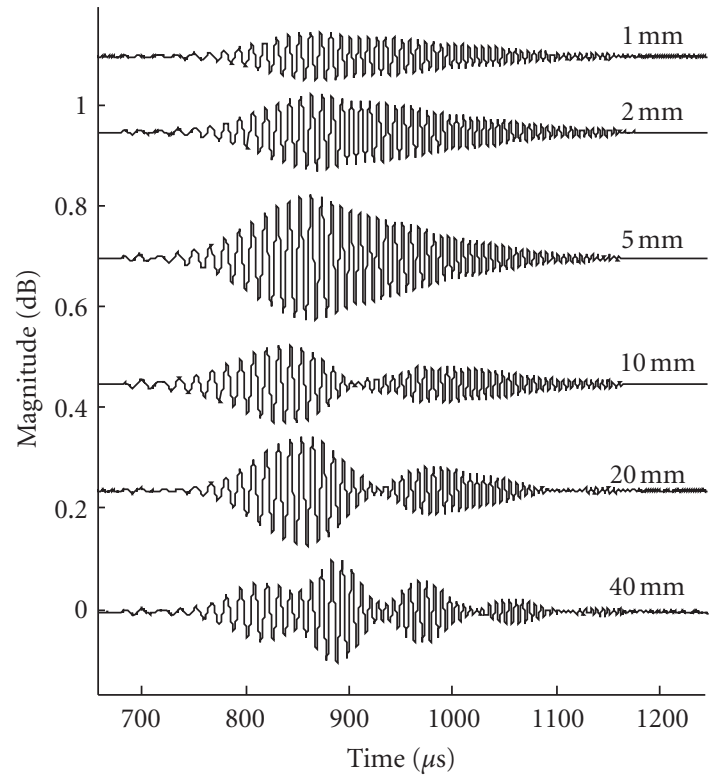

(a) polyvinylchloride (PVC)

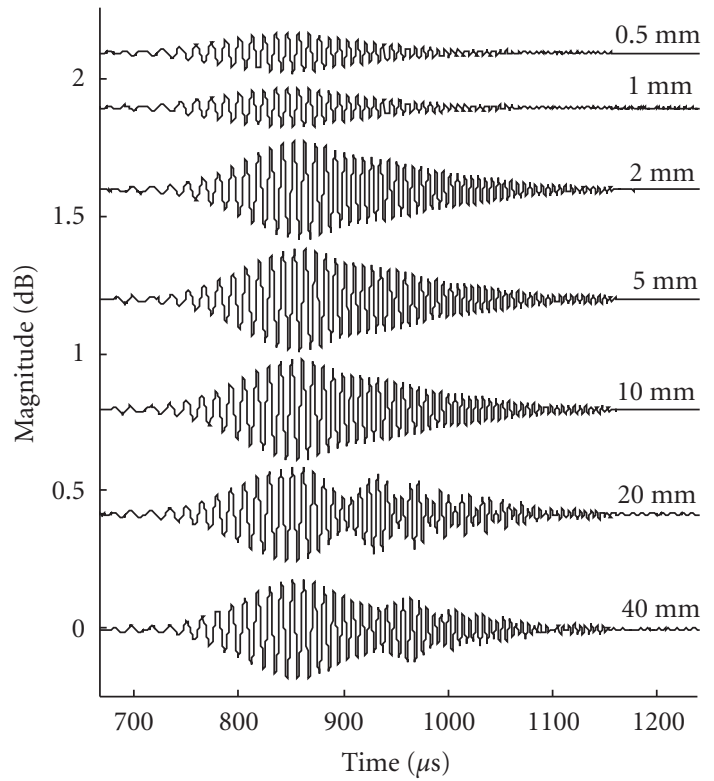

(b) brass

Figure 4: Time domain-reflected signals acquired for $40 \mathrm{~mm}$ thick targets.
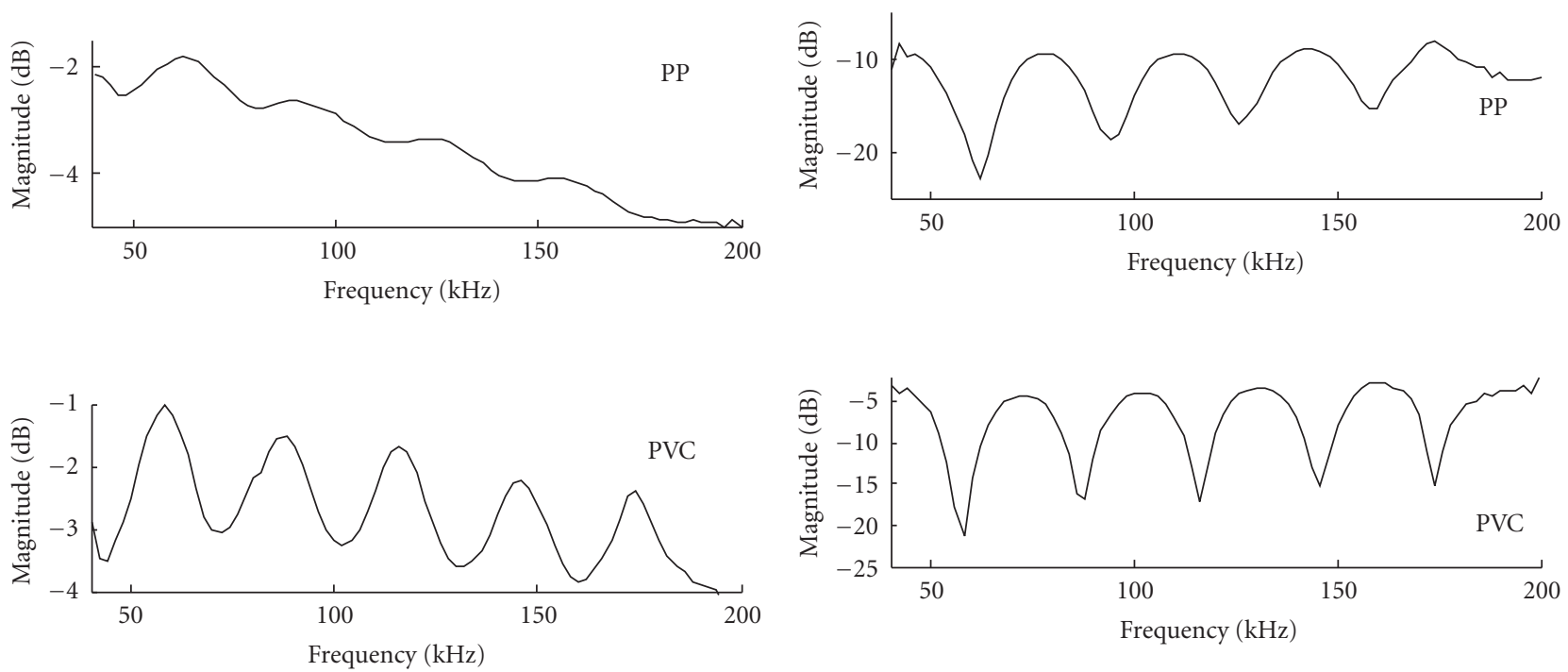

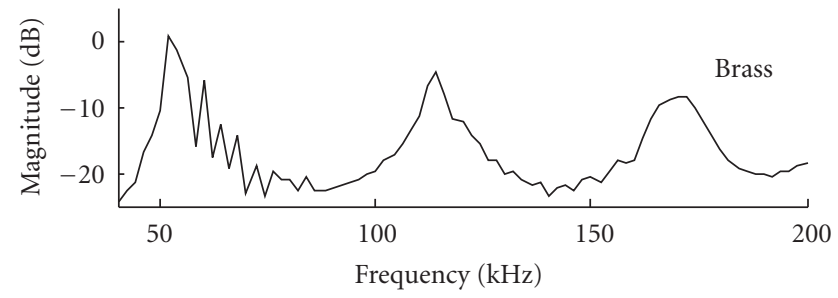

(a) transmission

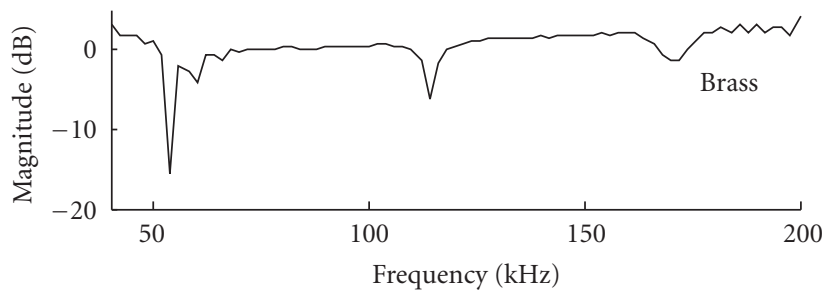

(b) pulse echo

Figure 5: Frequency domain spectra for polypropylene (PP), polyvinylchloride (PVC), and brass targets, obtained using the linear frequency-modulated (LFM) chirp signal. 


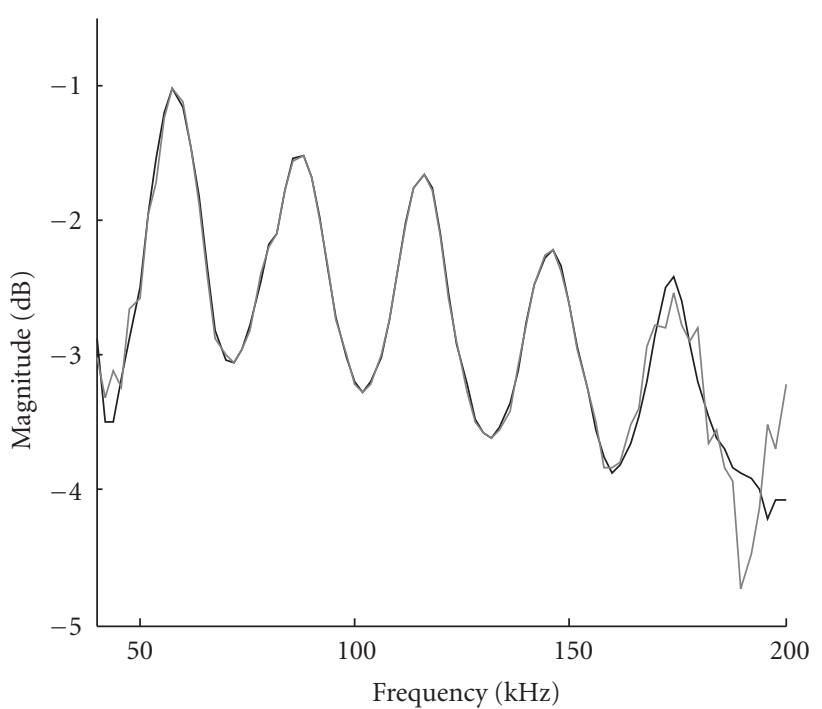

-LFM

$-\mathrm{ZPH}$

(a) transmission spectra

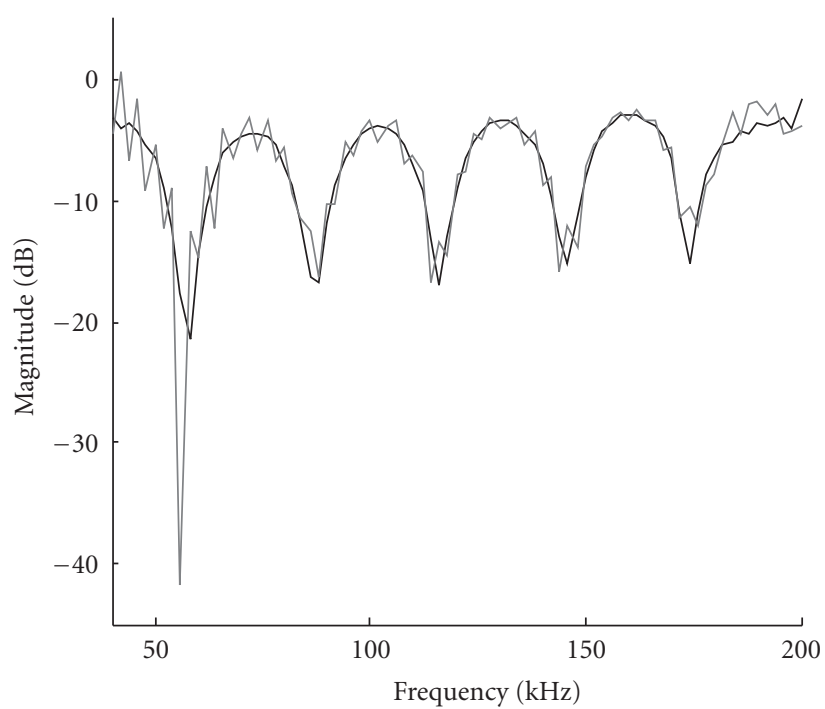

-LFM

$-\mathrm{ZPH}$

(b) pulse echo spectra

FIGURE 6: Frequency domain spectra for the $40 \mathrm{~mm}$ thick polyvinylchloride (PVC) target obtained using the zero phase ladder (ZPH) chirp (grey) and linear frequency modulated (LFM) chirp signal (black).

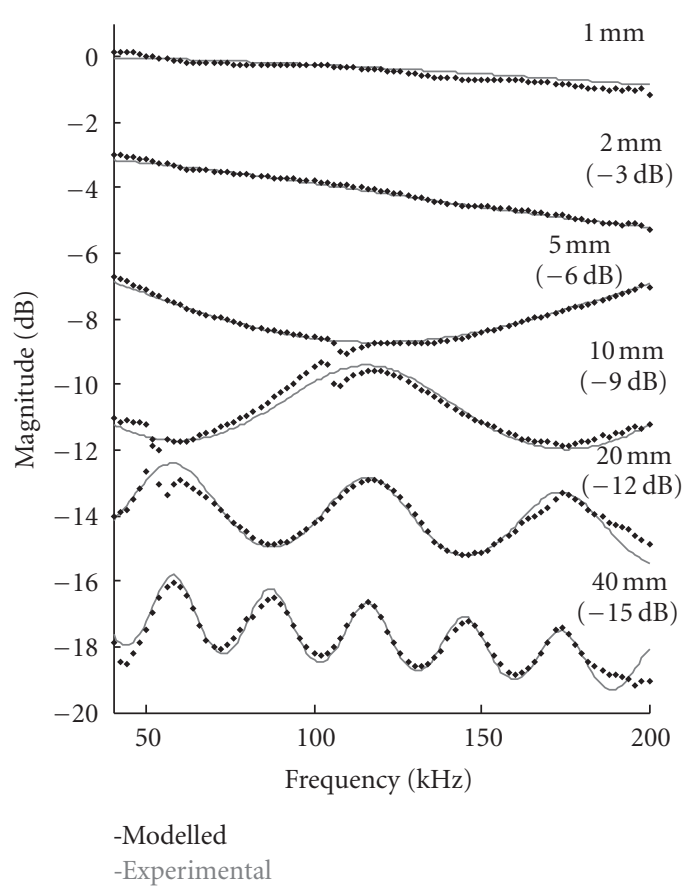

(a) transmission

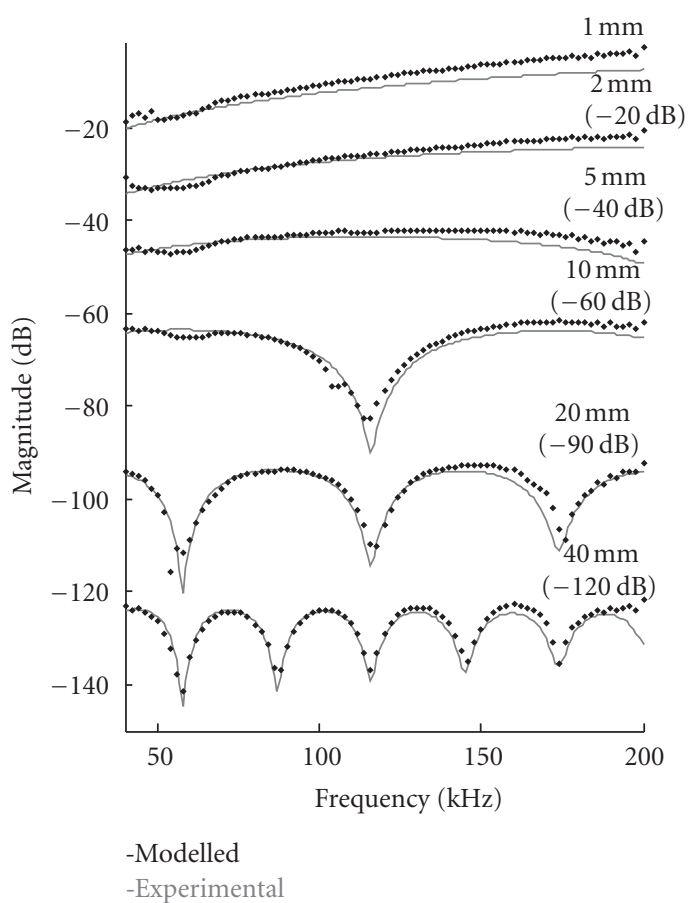

(b) pulse echo

FIGURE 7: Experimental (black diamonds) and modelled (grey lines) spectra for polypropylene (PP) targets of various thicknesses, obtained using the linear frequency modulated (LFM) chirp; traces are offset by the values shown in brackets.

agree with the modelled spectra for all thicknesses except $10 \mathrm{~mm}$ in transmission and $1 \mathrm{~mm}$ in reflection. The model parameters, determined by fitting the spectral response in the case of each $40 \mathrm{~mm}$ thick panel are used to predict the spectra for the other targets with knowledge of their thicknesses. The close fit of the predicted spectra gives confidence in the model parameters derived. The derived sound velocities and attenuation constants are shown in 


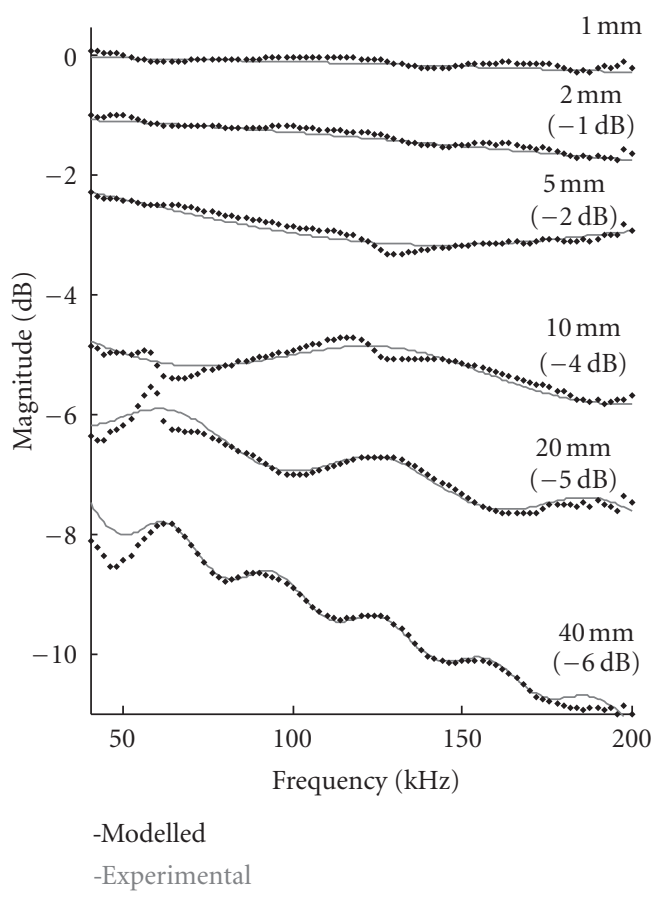

(a) Transmission

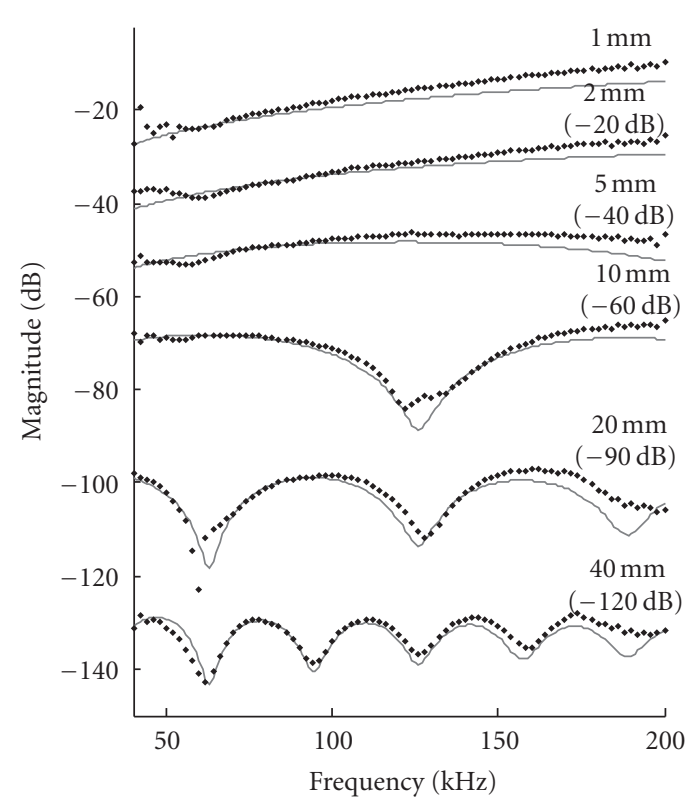

-Modelled

-Experimental

(b) pulse echo

FIGURE 8: Experimental (black diamonds) and modelled (grey lines) spectra for polyvinylchloride (PVC) targets of various thicknesses, obtained using the linear frequency modulated (LFM) chirp; traces are offset by the values shown in brackets.

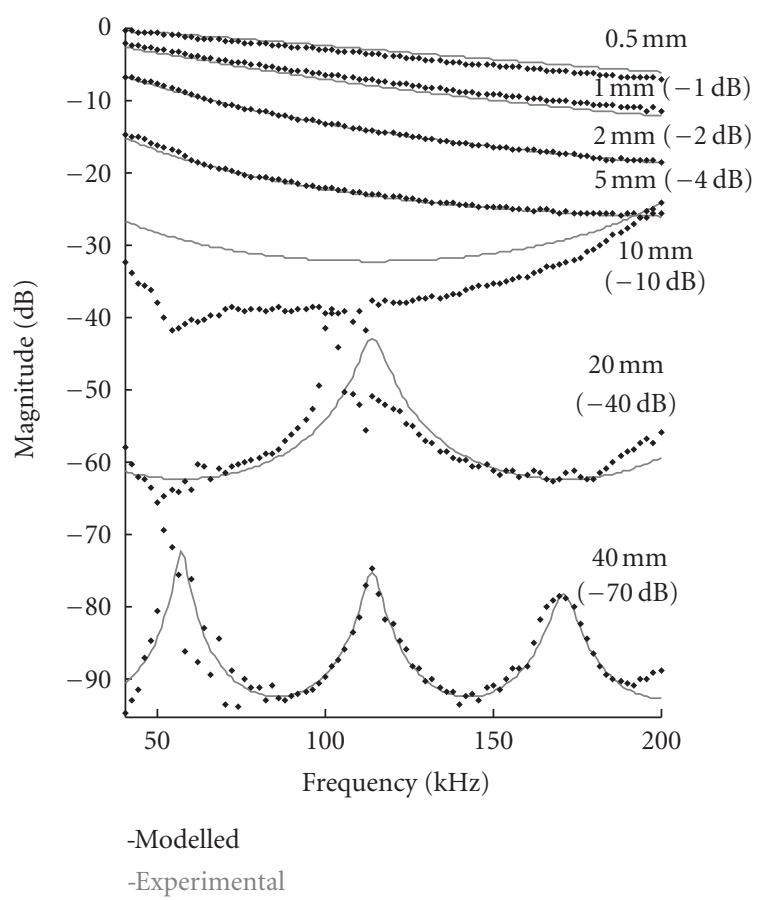

(a) transmission

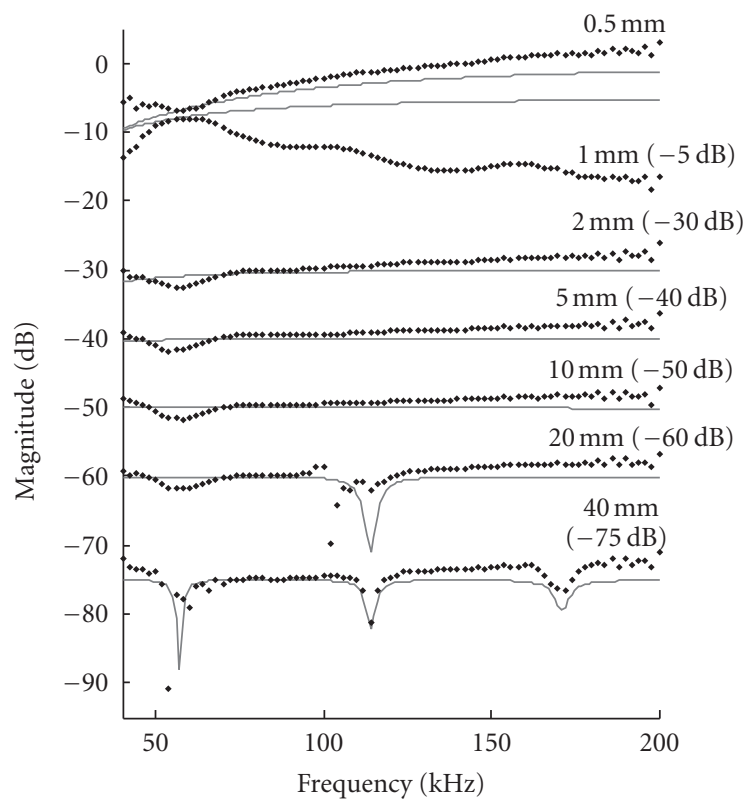

-Modelled

-Experimental

(b) pulse echo

FIGURE 9: Experimental (black diamonds) and modelled (grey lines) spectra for brass targets of various thicknesses, obtained using the linear frequency modulated (LFM) chirp; traces are offset by the values shown in brackets. 
TABLE 1

\begin{tabular}{|c|c|c|c|c|c|c|c|}
\hline \multirow[t]{2}{*}{ Material } & \multicolumn{2}{|c|}{ Reference } & \multicolumn{5}{|c|}{ Measured } \\
\hline & $\rho / \mathrm{kg} \mathrm{m}^{-3}$ & $c / \mathrm{ms}^{-1}$ & Z/Mrayl & $\% T$ & $\% R$ & $\rho / \mathrm{kg} \mathrm{m}^{-3}$ & $\Delta \rho$ (error) $/ \mathrm{kg} \mathrm{m}^{-3}$ \\
\hline Water & $998.1649[32]$ & $1482.955[33]$ & 1.48 & & & 996 & 1 \\
\hline Polypropylene (PP) & $902-906$ [34] & $2600[34]$ & 2.35 & 95 & 5 & 901 & 11 \\
\hline PVC & $1300-1400[34]$ & $2330[34]$ & 3.15 & 87 & 13 & 1441 & 19 \\
\hline Brass & $8400-8500$ [34] & $4372[34]$ & 36.94 & 15 & 85 & 8406 & 435 \\
\hline
\end{tabular}

TABLE 2

\begin{tabular}{lccc}
\hline Material & Sound velocity $\left(\mathrm{ms}^{-1}\right)$ & \multicolumn{2}{c}{ Attenuation coefficients $\left(\mathrm{Npm}^{-1} \mathrm{~Hz}^{-1}\right)$} \\
& & $a$ & $b$ \\
\hline PP & $2520 \pm 20$ & $(82 \pm 3) \times 10^{-6}$ & $(-73 \pm 7) \times 10^{-6}$ \\
PVC & $2320 \pm 20$ & $(30 \pm 3) \times 10^{-6}$ & $(15 \pm 7) \times 10^{-6}$ \\
Brass & $4560 \pm 20$ & $(6 \pm 3) \times 10^{-6}$ & $(68 \pm 7) \times 10^{-6}$ \\
\hline
\end{tabular}

TABLE 3

\begin{tabular}{lccc}
\hline Material & Sound velocity $\left(\mathrm{ms}^{-1}\right)$ & \multicolumn{2}{c}{ Attenuation coefficients $\left(\mathrm{Npm}^{-1} \mathrm{~Hz}^{-1}\right)$} \\
& & $a$ & $b$ \\
\hline PP & $2510 \pm 20$ & $(81 \pm 3) \times 10^{-6}$ & $(-64 \pm 7) \times 10^{-6}$ \\
PVC & $2330 \pm 20$ & $(31 \pm 3) \times 10^{-6}$ & $(10 \pm 7) \times 10^{-6}$ \\
Brass & $4540 \pm 20$ & $(7 \pm 3) \times 10^{-6}$ & $(41 \pm 7) \times 10^{-6}$ \\
\hline
\end{tabular}

Tables 2 and 3 for the LFM and ZPH signals, respectively. The errors, estimated by varying sound velocity and attenuation coefficients and assessing the fit, were less than $1 \%$ for sound velocity although somewhat higher for attenuation. Reference velocity values are shown in Table 1 . The calculated values are within $4.3 \%$, for PP and PVC and $4 \%$ for the brass. However, the reference values are only typical and the reference density values, also shown in Table 1, demonstrate that the material properties can vary significantly.

Although modelled and test spectra generally fit well, anomalies are observed in some spectra, that is, notches related to beating frequencies are observed in the spectra at $110 \mathrm{kHz}$ for 5 and $10 \mathrm{~mm} \mathrm{PP}, 130 \mathrm{kHz}$ for 5 and $10 \mathrm{~mm}$ PVC, and $110 \mathrm{kHz}$ for $20 \mathrm{~mm}$ brass. The thicknesses of these panels are approximately equal to $\lambda / 2$ or $\lambda / 4$ for the frequency at the notch. The notches are only observed for the panels, where the fundamental resonance frequency is within the bandwidth. It is not clear why these notches are observed; one explanation is that the resonating target induces resonances in the mounting structure. However, clear differences can be observed in the spectral signatures obtained for panels of different thickness, thus indicating the potential of the technique for thickness determination.

The effect of varying thickness in the model is shown in Figure 10. The modelled and test data are shown for thicknesses in the range 1 to $40 \mathrm{~mm}$, with predictions for $\pm 0.5 \mathrm{~mm}$. It is reasonable to suggest that thickness may be resolved to better than $\pm 0.5 \mathrm{~mm}$ using this technique.

\section{Discussion}

The fact that different materials of different thicknesses have diagnostic characteristics in relation to modulation in the time domain as well as associated spectral notches in the frequency domain demonstrates the ability of our method to discriminate both between materials and material thickness. The thickness resolution obtained using frequency domain spectra, is of the order of $\pm 0.5 \mathrm{~mm}$. Considering the wavelengths of our signals: $7.5 \mathrm{~mm}$ at $200 \mathrm{kHz}, 15 \mathrm{~mm}$ at $100 \mathrm{kHz}$, and $37.5 \mathrm{~mm}$ at $40 \mathrm{kHz}$, this gives resolutions of $\lambda / 15, \lambda / 30$, and $\lambda / 75$, respectively. It has been shown that dolphins can achieve resolutions of $\lambda / 50$ using similar frequency ranges [24], and, therefore, the resolution demonstrated in the present study is similar to that accredited in nature to dolphins.

Previous studies on underwater sonar have evaluated ER and IL for different materials in a similar frequency range [16-19]. Mikeska and Behrens [17] used frequencies of 50$500 \mathrm{kHz}$ using steps of $5 \mathrm{kHz}$. They determined that sound velocity estimations were accurate to $3 \%$ by varying sound speed in the model and comparing modelled results to test data. Barnard et al. [16] used discrete frequencies in the 100$500 \mathrm{kHz}$ range, with approximately $100 \mathrm{kHz}$ resolution, to compare modelled and test data for transmission coefficients with varying grazing angles. The fits of modelled to test data achieved were poorer than those demonstrated in the current study; however, Barnard et al. used multiple transducers to cover the frequency range, and measurements were made at discrete frequencies. The broadband nature and sensitivity of the devices in our work are thought to be responsible 


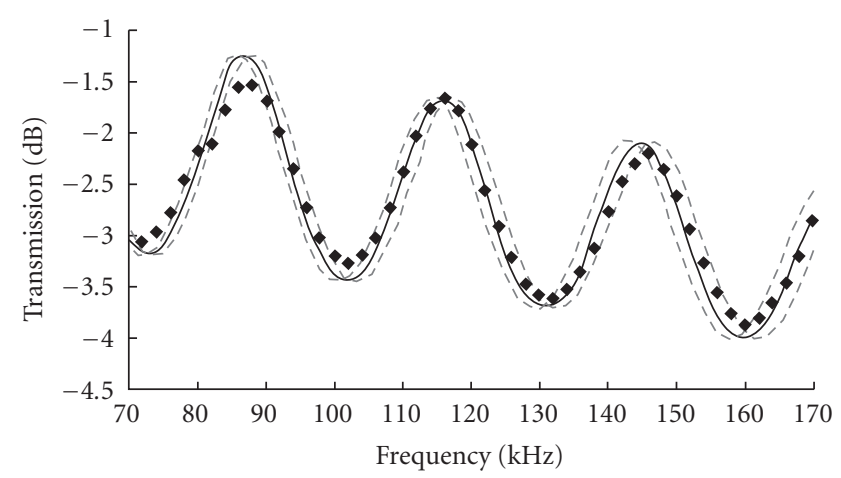

$\begin{array}{ll}- & \text { Exptl } \\ - & \text { Model } \\ --- & \text { Model } \pm 0.5 \mathrm{~mm}\end{array}$

(a) of $40 \mathrm{~mm}$ thickness

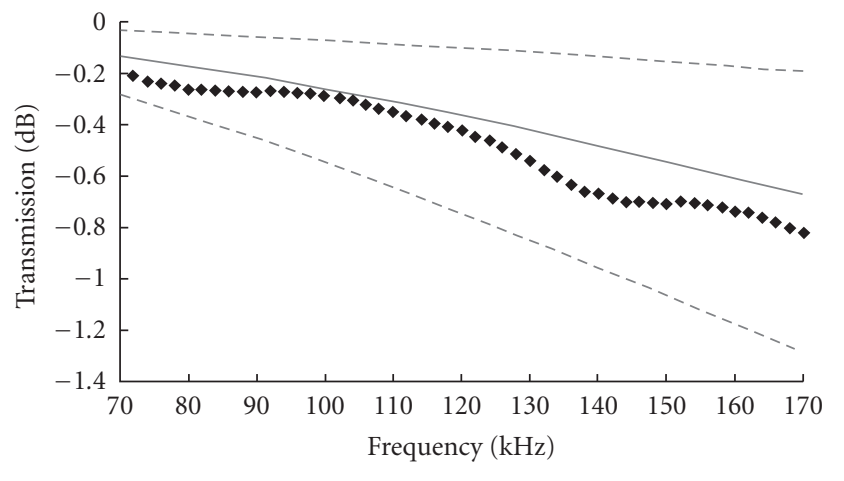

- Exptl

$\begin{array}{ll}- & \text { Model } \\ --- & \text { Model } \pm 0.5 \mathrm{~mm}\end{array}$

(b) $1 \mathrm{~mm}$ thickness

FIGURE 10: Experimental and modelled transmission spectra for polyvinylchloride (PVC) targets, with modelled data showing thickness variations of $\pm 0.5 \mathrm{~mm}$.

for the improved results. Thibieroz and Giangreco [18] used a $0-100 \mathrm{kHz}$ broadband pulse to interrogate a $30 \mathrm{~mm}$ thick steel panel. Perturbing phenomena affected the fit between modelled and test data, and this was due to diffraction effects and extraneous reflections. A signal processing technique was proposed to overcome these phenomena though some discrepancies were still observed-which were thought to be due to deviation from idealised plane wave insonification.

The performance of these waterborne applications can be compared to those in air, where thickness and sound velocity were determined using resonance spectra. Gan et al. [10] used a linear chirp signal with pulse compression for air-coupled measurements on brass and aluminium plates. Sound velocity of the plates was determined to within $1 \%$ (compared to a reference technique), and thicknesses were predicted from $2-10 \mathrm{~mm}$ with $4.7 \%$ error. The frequency range was $450 \mathrm{kHz}$ to $1.15 \mathrm{MHz}$, which represents a $\lambda$ range of $0.3-0.73 \mathrm{~mm}$, with the wavelength being significantly smaller than the thickness of the plates. The error for the $10 \mathrm{~mm}$ sample is therefore $0.47 \mathrm{~mm}$, which is in the middle of the $\lambda$ range used. The decreased performance could be caused by the poorer SNR attainable in air relative to that in water. Piezoelectric ultrasonic devices have been used in air-coupled applications, for instance, in determining pipe thicknesses [9]. Resonance spectra obtained using tone bursts with a $500 \mathrm{kHz}$ frequency were used to determine thicknesses of steel, quartz, and glass of $17-50 \mathrm{~mm}$ thickness with $<5 \%$ error. The wavelength at $500 \mathrm{kHz}$ is $0.7 \mathrm{~mm}$ was smaller than the sample thickness, and less than the errors $(0.85-2.5 \mathrm{~mm})$. Previous studies on immersion testing have used higher frequencies to obtain signals reflected from the front and back face of targets. Broadband pulses were used in order to determine parameters such as phase velocity, sample thickness and attenuation [1,3-5]. The resolutions found using our method are similar to those obtained with higher frequencies; therefore, while using lower frequencies, we are getting comparable performance to that of higher frequencies. This is advantageous when dealing with highly attenuating materials.

These results demonstrate the successful application for material characterisation of a LFM chirp signal, optimised in terms of frequency resolution and signal length, to reduce extraneous reflections, with a Tukey window. The sound velocity and attenuation measurement performance of the $\mathrm{ZPH}$ signals is comparable to that of LFM signals, but has a poorer SNR. However, ZPH signals offer significant potential for usage in low-frequency applications as they allow greater resolution in measurement. These signals, similar to dolphin clicks, could prove to be advantageous when shorter time duration signals are required and amplification could be used to overcome the SNR issue. Moreover, utilising phase information of this signal could potentially be important and is an area currently under investigation.

\section{Conclusion}

A new method is described that enables ultrasonic material characterisation to be performed in the $40-200 \mathrm{kHz}$ range. The frequency range is inspired by biological systems and differs from the more commonly used frequency ranges of $\mathrm{MHz}$ frequencies for materials testing and low $\mathrm{kHz}$ frequencies for sonar applications. This novel method differs from previously published studies for assessing material discrimination and thickness resolution. Although bioinspired, this method does not attempt to copy the echolocation mechanisms of biological systems. Instead it uses the frequency range, and broadband signal attributes as inspiration in defining the signals used. The study shows material discrimination is possible based on both temporal and spectral results. The thickness resolution is comparable with that of bats and dolphins and is achieved using wavelengths much greater than previous studies. The findings have significant impact in areas such as NDTE, medical physics, and geophysics, where improved resolution is sought 
using lower frequencies to overcome the problem of highly attenuating media.

\section{Acknowledgments}

This paper was undertaken as part of the Biologically Inspired Acoustic Systems (BIAS) project funded by RCUK via the Basic Technology Programme Grant reference number EP/C523776/1. The BIAS project involved collaboration between the British Geological Survey, Leicester University, Fortkey Ltd., Southampton University, Leeds University, Edinburgh University, and Strathclyde University. This paper is published under the permission of the Executive Director of the British Geological Survey (NERC).

\section{References}

[1] R. A. Kline, "Measurement of attenuation and dispersion using an ultrasonic spectroscopy technique," Journal of the Acoustical Society of America, vol. 76, no. 2, pp. 498-504, 1984.

[2] F. Peters and L. Petit, "Propagation of ultrasound waves in concentrated suspensions," Acustica, vol. 86, no. 5, pp. 838$846,2000$.

[3] P. He, "Measurement of acoustic dispersion using both transmitted and reflected pulses," Journal of the Acoustical Society of America, vol. 107, no. 2, pp. 801-807, 2000.

[4] D. K. Hsu and M. S. Hughes, "Simultaneous ultrasonic velocity and sample thickness measurement and application in composites," Journal of the Acoustical Society of America, vol. 92, no. 2, pp. 669-675, 1992.

[5] R. Raišutis, R. Kažys, and L. Mažeika, "Application of the ultrasonic characterization methods for highly attenuating plastic materials," NDT and E International, vol. 40, no. 4, pp. 324-332, 2007.

[6] A. I. Lavrentyev and S. I. Rokhlin, "Determination of elastic moduli, density, attenuation, and thickness of a layer using ultrasonic spectroscopy at two angles," Journal of the Acoustical Society of America, vol. 102, no. 6, pp. 3467-3477, 1997.

[7] A. I. Lavrentyev and S. I. Rokhlin, "Anomalous attenuation effect on reflectivity of an ultrasonic wave from a thin layer between dissimilar materials," Journal of the Acoustical Society of America, vol. 101, no. 6, pp. 3405-3414, 1997.

[8] H. J. McSkimin and P. Andreatch Jr., "A water immersion technique for measuring attenuation and phase velocity of longitudinal waves in plastics," Journal of the Acoustical Society of America, vol. 49, no. 3, pp. 713-722, 1971.

[9] S. Whiteley, G. Hayward, and D. Reilly, "Thickness measurement using air-coupled ultrasound," Insight, vol. 39, no. 11, pp. 770-775, 1997.

[10] T. H. Gan, D. A. Hutchins, D. R. Billson, and D. W. Schindel, "The use of broadband acoustic transducers and pulsecompression techniques for air-coupled ultrasonic imaging," Ultrasonics, vol. 39, no. 3, pp. 181-194, 2001.

[11] P. Pallav, T. H. Gan, and D. A. Hutchins, "Elliptical-Tukey chirp signal for high-resolution, air-coupled ultrasonic imaging," IEEE Transactions on Ultrasonics, Ferroelectrics, and Frequency Control, vol. 54, no. 8, pp. 1530-1540, 2007.

[12] R. J. Bobber, Underwater Electroacoustic Measurements, Peninsula Publishing, Los Altos, Calif, USA, 1988.

[13] V. F. Humphrey, S. P. Robinson, J. D. Smith et al., "Acoustic characterization of panel materials under simulated ocean conditions using a parametric array source," Journal of the
Acoustical Society of America, vol. 124, no. 2, pp. 803-814, 2008.

[14] J. C. Piquette, "Some new techniques for panel measurements," Journal of the Acoustical Society of America, vol. 100, no. 5, pp. 3227-3236, 1996.

[15] D. H. Trivett and A. Z. Robinson, "Modified Prony method approach to echo-reduction measurements," Journal of the Acoustical Society of America, vol. 70, no. 4, pp. 1166-1175, 1981.

[16] G. R. Barnard, J. L. Bardin, and J. W. Whiteley, "Acoustic reflection and transmission characteristics for thin plates," Journal of the Acoustical Society of America, vol. 57, no. 3, pp. 577-584, 1975.

[17] E. E. Mikeska and J. A. Behrens, "Evaluation of transducer window materials," Journal of the Acoustical Society of America, vol. 59, no. 6, pp. 1294-1298, 1976.

[18] T. Thibieroz and C. Ginagreco, "Impulse acoustical measurements of the transmission coefficients of immersed panels," Journal of the Acoustical Society of America, vol. 4, pp. 215-236, 1991.

[19] V. F. Humphrey, "The measurement of acoustic properties of limited size panels by use of a parametric source," Journal of Sound and Vibration, vol. 98, no. 1, pp. 67-81, 1985.

[20] T. Misaridis and J. A. Jensen, "Use of modulated excitation Signals in medical ultrasound. Part I: basic concepts and expected benefits," IEEE Transactions on Ultrasonics, Ferroelectrics, and Frequency Control, vol. 52, no. 2, pp. 177-191, 2005.

[21] M. Gutowski, J. Bull, T. Henstock et al., "Chirp sub-bottom profiler source signature design and field testing," Marine Geophysical Researches, vol. 23, no. 5-6, pp. 481-492, 2002.

[22] C. Capus, Y. Pailhas, K. Brown, D. M. Lane, P. W. Moore, and D. Houser, "Bio-inspired wideband sonar signals based on observations of the bottlenose dolphin (Tursiops truncatus)," Journal of the Acoustical Society of America, vol. 121, no. 1, pp. 594-604, 2007.

[23] W. W. L. Au and J. A. Simmons, "Echolocation in dolphins and bats," Physics Today, vol. 60, no. 9, pp. 40-45, 2007.

[24] W. W. L. Au and D. A. Pawloski, "Cylinder wall thickness difference discrimination by an echolocating Atlantic bottlenose dolphin," Journal of Comparative Physiology A, vol. 170, no. 1, pp. 41-47, 1992.

[25] W. W. L. Au and C. W. Turl, "Material composition discrimination of cylinders at different aspect angles by an echolocating Dolphin," Journal of the Acoustical Society of America, vol. 89, no. 5, pp. 2448-2451, 1991.

[26] W. W. L. Au, K. J. Benoit-Bird, and R. A. Kastelein, "Modeling the detection range of fish by echolocating bottlenose dolphins and harbor porpoises," Journal of the Acoustical Society of America, vol. 121, no. 6, pp. 3954-3962, 2007.

[27] P. E. Nachtigall, W. W. L. Au, H. L. Roitblat, and J. L. Pawloski, "Dolphin biosonar: a model for biomimetic sonars," in Proceedings of the 1st International Symposium on Aqua Bio Mechanisms, pp. 115-121, 2000.

[28] W. W. L. Au, The Sonar of Dolphins, Springer, New York, NY, USA, 1993.

[29] T. Imaizumi, M. Furusawa, T. Akamatsu, and Y. Nishimori, "Measuring the target strength spectra of fish using dolphinlike short broadband sonar signals," Journal of the Acoustical Society of America, vol. 124, no. 6, pp. 3440-3449, 2008.

[30] I. Matsuo, T. Imaizumi, T. Akamatsu, M. Furusawa, and Y. Nishimori, "Analysis of the temporal structure of fish echoes using the dolphin broadband sonar signal," Journal of the Acoustical Society of America, vol. 126, no. 1, pp. 444-450, 2009.

[31] G. L. Gooberman, Ultrasonics: Theory and Application, The 
English Universities Press, London, UK, 1968.

[32] D. R. Lide, CRC Handbook of Chemistry and Physics, CRC Press, Boca Raton, Fla, USA, 76th edition, 1995.

[33] V. A. Del Grosso and C. W. Mader, "Speed of sound in pure water," Journal of the Acoustical Society of America, vol. 52, no. 5, pp. 1442-1446, 1972.

[34] G. W. C. Kaye, Tables of Physical and Chemical Constants and Some Mathematical Functions, Longman, London, UK, 16th edition, 1995. 